\title{
Correction to: A p (•)-Poincaré-type inequality for variable exponent Sobolev spaces with zero boundary values in Carnot groups
}

\author{
Thomas Bieske $^{1}$ (D) $\cdot$ Robert D. Freeman ${ }^{1}$ \\ Published online: 3 January 2019 \\ ○) Springer Nature Switzerland AG 2019
}

\section{Correction to: Anal.Math.Phys. (2018) 8:289-308 https://doi.org/10.1007/s13324-018-0235-7}

In this short note, we remark on the main theorem of [1, Theorem 5.1]. First, the authors would like to thank the referee for pointing out that a more general result related to [1, Theorem 5.1], was proved for vector fields satisfying Hörmander's condition by $\mathrm{Li}$ et al. [3, Theorem 1.10]. In [3, Theorem 3.1], the authors first show a more general result for boundedness of fractional integral operators in variable Lebesgue spaces on spaces of homogeneous type and combine this with a representation formula proved in [2] to establish a higher order Poincaré inequality for vector fields satisfying Hörmander's condition on Boman chains (and therefore metric balls). Here they took the assumptions that $\mathrm{p}(\cdot)$ is log-Hölder continuous and $1<\mathrm{p}^{-} \leq \mathrm{p}^{+}<Q / m$, where $Q$ is the homogenous dimension and $m$ is the dimension of the variable exponent space (in our case $m=1$ ).

However, the main theorem [1, Theorem 5.1] does not follow directly from [3, Theorem 1.10]. The difference is in the assumption that $\mathrm{p}(\cdot)$ is $\log$-Hölder continuous in $[3$, Theorem 1.10] while [1, Theorem 5.1] requires that the variable exponent $\mathrm{p}(\cdot)$ satisfies the jump condition. This allows for the case where $\mathrm{p}^{-}>Q$ in the gauge balls discussed in Section 5 of [1]. Thus, the condition $1<\mathrm{p}^{-} \leq \mathrm{p}^{+}<Q$ in [3, Theorem 1.10] is violated. Consequently, when $\mathrm{p}^{-}>Q$, then the hypotheses of $[3$, Theorem 1.10] do not hold but those of [1, Theorem 5.1] do hold.

Furthermore, the main theorem [1, Theorem 5.1] does not follow from combining [3, Theorem 3.1] and the representation formula of functions with boundary value

The original article can be found online at https://doi.org/10.1007/s13324-018-0235-7.

\footnotetext{
$\bowtie \quad$ Thomas Bieske

tbieske@usf.edu

Robert D. Freeman

rfreeman1@mail.usf.edu

1 Department of Mathematics and Statistics, University of South Florida, Tampa, FL 33620, USA
} 
zero for vector fields established in [4]. Again, we have the possibility that $\mathrm{p}^{-}>Q$ in gauge balls but this fails the conditions in [3, Theorem 3.1].

In both cases, it is obvious that if $\mathrm{p}^{+} \leq \frac{Q \mathrm{p}^{-}}{Q-\mathrm{p}^{-}}$in gauge balls, then we have the jump condition satisfied and this also agrees with the conditions in [3, Theorem 1.10] and thus [1, Theorem 5.1] does indeed follow directly from [3, Theorem 1.10]. Similarly, this is also true for [3, Theorem 3.1] and we could use representation formulas in [4] to easily obtain [1, Theorem 5.1].

\section{References}

1. Bieske, T., Freeman, R.D.: A $p(x)$-Poincaré-type inequality for variable exponent Sobolev spaces with zero boundary values in Carnot groups. Anal. Math. Phys. 8, 289-308 (2018)

2. Franchi, B., Lu, G., Wheeden, R.L.: Representation formulas and weighted Poincaré inequalities for Hörmander vector fields. Ann. Inst. Fourier (Grenoble) 45, 577-604 (1995)

3. Li, X., Lu, G.Z., Tang, H.L.: Poincaré and Sobolev inequalities for vector fields satisfying Hörmander's condition in variable exponent Sobolev spaces. Acta Math. Sin. Engl. Ser. 31(7), 1067-1085 (2015)

4. Lu, G.: Weighted Poincaré and Sobolev inequalities for vector fields satisfying Hörmander's condition and applications. Rev. Mat. Iberoam. 8, 367-439 (1992)

Publisher's Note Springer Nature remains neutral with regard to jurisdictional claims in published maps and institutional affiliations. 